\title{
Influencia social online: nuevas tecnologías en la comunicación del sector turístico
}

\section{Online social influence: new technologies in hospitality communication}

\author{
Diana Gavilan \\ Profesor Contratado Doctor Departamento de Comercialización \\ e Investigación de Mercados \\ (Universidad Complutense de Madrid) \\ Gema Martínez-Navarro \\ Profesor Ayudante Doctor Departamento de Comercialización \\ e Investigación de Mercados \\ (Universidad Complutense de Madrid) \\ Susana Fernández-Lores \\ Profesor Asociado Departamentos de Comunicación e Investigación \\ de Mercados y Métodos Cuantitativos \\ (ESIC, Business \& Marketing School)
}

Fecha de recepción: 29 de mayo de 2017

Fecha de revisión: 19 de junio de 2017

Fecha de publicación: 1 de julio de 2017

Para citar este artículo: Gavilan, D., Martínez-Navarro, G. y Fernández-Lores, S. (2017):

Influencia social online: nuevas tecnologías en la comunicación del sector turístico, Icono 14, volumen 15 (2), pp. 138-167. doi: 10.7195/ri14.v15i2.1065 
MONOGRÁFICO

\section{Resumen}

Internet ha revolucionado el sector turístico desde el punto de vista del consumidor y la empresa. La información actualmente es abundante y accesible y el viajero, cada vez más tecnológico, se ha convertido en prescriptor. Así, evaluaciones y comentarios hechos por usuarios anónimos en los sites de alojamientos representan una forma de influencia de gran impacto en la toma de decisiones que ha transformado los tradicionales modelos de comunicación de las empresas. Este trabajo estudia la influencia social que ejercen las valoraciones numéricas y los comentarios en el proceso de elección de alojamientos turísticos, teniendo en cuenta la susceptibilidad del propio usuario a la información que procede de otras personas. Para ello se ha realizado un experimento cuyas conclusiones revelan que las evaluaciones constituyen un factor crítico en la decisión, pero están supeditadas al volumen de comentarios y que la susceptibilidad del usuario a la influencia social modera este proceso.

Palabras clave: Evaluaciones numéricas - Comentarios - Decisiones del consumidor Comunicación online - Influencia social - Turismo

\section{Abstract}

Internet has shaken up the tourism sector from the point of view of the consumer and of the company. Information is currently abundant and accessible; the traveler is more technological and has become an influencer. Thereby, ratings and reviews made by anonymous users on hospitality websites represent a form of influence with great impact on decision making that has transformed companies' traditional communication models. This paper studies the mechanisms of social influence, specifically the role played by ratings and reviews in the choice of a tourist accommodation, taking into account the susceptibility of the user to the information that comes from others. An experiment was carried out. Conclusions reveal that ratings do constitute a critical factor in the decision, but are contingent upon the volume of reviews and that individual susceptibility to social influence moderated this process.

Key Words: Ratings - Reviews - Customer decisions - Online communication - Social influence - Hospitality 


\section{Introducción}

La aparición de internet y el auge de las redes sociales han supuesto un importante cambio en la forma en la que los usuarios organizan y deciden los viajes. La reserva de un vuelo o un alojamiento nos lleva a buscar y compartir experiencias vividas por otros, que adoptan la forma de valoraciones y comentarios, para orientar nuestras opciones de elección.

Según los datos de la web de gestión de reputación de alojamientos Olery.com, el $81 \%$ de los viajeros considera importantes los comentarios sobre los hoteles a la hora de hacer una reserva de alojamiento. El $46 \%$ de los usuarios publica comentarios y lo que puede ser más importante: el $49 \%$ de los viajeros no reservaría en un hotel sin comentarios.

Estos datos sugieren que nuestra sociedad confía cada vez más en las opiniones emitidas por desconocidos en la red. El entorno virtual ha adquirido un papel relevante para el consumidor a la hora de buscar información acerca de un producto o servicio y de facilitar su elección de compra (Shim, Eastlick, Lotz, y Warrington, 2001). El número de consumidores que recurren a internet para obtener información y recomendaciones acerca de productos y servicios es inmenso y tiende a ser cada vez mayor (Peterson y Merino, 2003).

Las empresas son conscientes de que el desarrollo del ciberespacio conlleva emparejado el fenómeno de influencia social online que plantea innumerables retos: cómo captar, analizar, interpretar y gestionar la participación de los usuarios (Litvin, Goldsmith y Pan, 2008). Las ciencias sociales reconocen que las personas tienen la habilidad de influir unas sobre otras (Ditcher, 1966); de hecho la influencia de los comentarios de otros en las decisiones de compra ha sido probada en diversos estudios sobre productos como libros (Chevalier y Mayzlin, 2006), estancias en hoteles (Vermeulen y Seegers, 2008; Zhu y Zhang, 2009) o películas de cine (Reinstein y Snyder, 2005). También se ha contrastado la capacidad que tienen los comentarios para modificar la notoriedad de un producto (Duan, Gu y Whinston, 2008), su reputación, la de su marca o la percepción de confiabilidad (Lee, Shi, Cheung, Lim y Ling, 2011). Incluso, los comentarios de otros han demostrado ser 


\section{MONOGRÁFICO}

un punto de referencia desde el que enfocar la experiencia de consumo (Moe y Schweidel, 2011).

Sin embargo, internet es un entorno dinámico donde el incremento en el volumen de información generado y distribuido por los propios consumidores conduce a la adopción de nuevas formas de buscar y de acceder a esa información para utilizarla en futuras decisiones. En este contexto, las evaluaciones numéricas y los comentarios hechos por usuarios anónimos a través de las plataformas tecnológicas se han convertido en una de las fuentes de información más utilizadas ya que reducen el riesgo, facilitan la toma de decisiones y permiten la interacción entre quienes comparten intereses (Amblee y Bui, 2011; Sparks y Browning, 2011; Lee, Law y Murphy, 2011).

Teniendo como marco de referencia este entorno, nuestro trabajo tiene como objetivo general analizar la influencia que tienen las valoraciones numéricas y los comentarios de otros usuarios en la elección online de un alojamiento turístico y por tanto, las implicaciones que esta influencia tiene para las empresas.

El trabajo se divide en cinco apartados: una revisión de la literatura sobre la influencia social online, con referencia específica al ámbito de la contratación de alojamientos turísticos; un análisis de la situación y tráfico de los sites de alojamientos turísticos; material y métodos empleados en el diseño del experimento; exposición de resultados y por último, discusión.

\subsection{La influencia social online}

A priori, la información que facilitan los sites de alojamientos turísticos en forma de evaluaciones y comentarios hechos por otros usuarios podría ser considerada una fuente de información de utilidad limitada. Esto se debe a varias cuestiones. Por un lado, las opiniones vertidas solo representan las preferencias de otros usuarios, y no un análisis exhaustivo del producto o servicio evaluado; además los autores de la información no proceden de una muestra representativa de usuarios, generalmente son los usuarios extremadamente satisfechos o insatisfechos los que se inclinan a ser más participativos evaluando y comentando (Anderson, 1998) 
y por otro lado, hay que considerar la posibilidad de que las partes interesadas pueden manipular estos contenidos ensalzando las cualidades y virtudes de sus productos (Dellarocas y Narayan, 2006).

Hechas estas consideraciones, en virtud de las cuales los usuarios deberían desoír las evaluaciones y comentarios de los otros, se constata lo contrario. Año tras año la influencia y la confianza en las evaluaciones y comentarios publicados en la red crece (BrightLocal, 2014). La capacidad adquirida por los ciudadanos para participar e influir en determinados aspectos de la realidad que les rodea es una de las características más relevantes y que han impactado de forma más notable en el éxito de los negocios (Parra, Bulchand, Gutiérrez y Díaz, 2010). Este fenómeno de influencia social supone la aceptación de información o consejo de personas que no son conocidas por el sujeto pero que proporcionan evidencias creíbles de la realidad (Burnkrant y Cousineau, 1975). Los usuarios confían en las evaluaciones $\mathrm{y}$ los comentarios hechos por otros usuarios en la red.

La influencia social se vuelve especialmente importante cuando los sujetos se enfrentan a procesos de decisión en los que confluyen varias circunstancias: restricción de tiempo, conocimiento limitado, riesgo percibido y/o falta de interés por adoptar una decisión con esfuerzo (Lee et al., 2011). En este escenario observar qué han hecho otros y acceder a sus experiencias, aunque sean desconocidos, sirve de ayuda en el proceso de decisión, adquiriendo especial relevancia.

En internet, para adoptar una decisión de compra, el usuario se enfrenta a una enorme cantidad de información que puede resultarle difícil de procesar. Por ello tanto las evaluaciones numéricas (puntuaciones en escalas de 5 o de 10 posiciones), como el volumen de comentarios (que sugiere a priori el número de personas que avalan la opción) proporcionan una información fácil de entender y rápida de procesar que agiliza las decisiones. La información expresada en forma de números ayuda a cribar ofertas y/o perfilar alternativas.

Confiamos en las evaluaciones numéricas y las consideramos información solvente (Nielsen, 2012). Esta confianza se extiende hasta la disponibilidad de pago: los consumidores están dispuestos a pagar más por productos con una valoración 


\section{MONOGRÁFICO}

"excelente", numéricamente 5, que con una valoración "buena", numéricamente 4, lo que no pasa desapercibido para las empresas que las traducen en precios superiores a la media -premium- (ComScore, 2007).

Sin embargo, para comprender la influencia social que ejercen las evaluaciones y comentarios también debe tenerse en cuenta el grado de susceptibilidad del sujeto a esta forma de influencia social. Nos referimos a un rasgo de personalidad que varía entre individuos (Bearden, Netemeyer y Teel, 1989) y explica la mayor o menor sensibilidad del usuario a prestar atención y apoyarse en las opiniones y la conducta de otros usuarios (evaluaciones numéricas y comentarios) en sus decisiones de compra.

\subsection{Influencia social en la contratación de alojamientos turísticos}

El mercado turístico español lidera el ranking mundial de competitividad turística según datos del Foro Económico Mundial (FEM), organismo que analiza la industria vacacional de 141 países de todo el mundo. En su informe de 2015 se señala que el turismo español ocupa una de las primeras posiciones en el podio internacional gracias a la riqueza de sus recursos culturales, sus infraestructuras y la adaptación a los hábitos de consumo digitales. Estamos por tanto, ante un mercado relevante y en expansión en el que los usuarios se enfrentan a procesos de decisión susceptibles de ser investigados con un enfoque académico.

Cada año cientos de miles de potenciales usuarios de alojamientos turísticos consultan sites de alojamientos. PhoCusWright (2013) estima que a nivel mundial, la influencia que ejercen las evaluaciones y comentarios podría representar hasta 10.000 millones de dólares para el sector turístico. Nos encontramos por lo tanto en un ámbito especialmente sensible a la influencia social.

Por otro lado, debe considerarse que el producto turístico tiene una naturaleza experiencial y es intangible, es decir, no puede ser evaluado antes de su consumo, lo que hace que muchos productos hoteleros y turísticos se perciban como compras de alto riesgo (Litvin, Goldsmith y Pan, 2008) para las cuales, la experiencia de otros usuarios adquiere gran valor a la hora de tomar una decisión de compra. Este 
hecho, unido a las numerosas alternativas de alojamientos que el usuario puede encontrar online, le llevan a buscar opiniones que gocen del respaldo de otros antes de concretar su decisión con el objetivo de reducir el riesgo del fracaso y evitar el coste de experimentar por uno mismo (Rao, Greve y Davis, 2001). La evaluación numérica del alojamiento y los comentarios de otros usuarios sobre su experiencia proporcionan pistas confiables de cómo es la realidad de cada opción, facilitando un proceso de persuasión de tipo periférico (Salmon et al., 2015).

La influencia social online tiene lugar en los procesos de decisión de compra de numerosos productos (libros, dispositivos tecnológicos,...) pero es de especial relevancia en la contratación de servicios de ocio, y concretamente en alojamientos turísticos (Olabarri, Monge y Usín, 2015). Algunos estudios han puesto de manifiesto la importancia que tienen las evaluaciones y los comentarios de los usuarios en las decisiones de compra (Gutiérrez-Taño, Bulchand, Díaz y Parra, 2013; Martínez, Bernal y Mellinas, 2012) así como los factores que impulsan a los usuarios a proporcionar estos contenidos (Serra y Salvi, 2014). Incluso hay trabajos como el de Tuominen (2011) que con los datos Tripadvisor ha tratado de establecer una relación entre las valoraciones numéricas, el volumen de comentarios y los resultados del hotel en términos de nivel de ocupación y precio medio. En esta línea del impacto económico, otras investigaciones han observado cómo las opiniones positivas pueden incrementar significativamente el número de reservas de habitaciones de un alojamiento (Ye, Law y Gu, 2009); y cómo la publicación en los sites de alojamientos turísticos de valoraciones numéricas y comentarios de clientes puede servir para cambiar sus precios (Yacouel y Fleischer, 2010; Ögüt y Onur, 2012; Diana-Jens y Rodríguez, 2015).

Estos trabajos ponen de manifiesto que las evaluaciones y comentarios realizados en los sites de alojamientos turísticos son una forma eficaz de incrementar la relevancia de un alojamiento, el nivel de ocupación o el precio medio. Sin embargo, no se han analizado en detalle los mecanismos que conducen a ese resultado, y por lo tanto la influencia que ejercen las evaluaciones y los comentarios en diferentes aspectos del proceso de decisión: atención suscitada por una oferta, estimación de la capacidad informativa de ésta, interés que despierta o probabilidad de reserva. Igualmente es necesario si no se quiere ofrecer una explicación sesgada, conside- 
rar en este proceso el papel que pueda desempeñar el grado de susceptibilidad del propio usuario a la influencia social online, cuestiones todas ellas objeto de este trabajo.

\section{Situación y tráfico de los sites de alojamientos turísticos}

Con objeto de entender y dimensionar correctamente el panorama de los sites de alojamientos turísticos, ofrecemos una breve descripción de la situación y tráfico de estos sites, aplicando las herramientas de analítica web Alexa y Semrush.

En primer lugar se han identificado los principales sites del sector, gracias al análisis de las palabras clave y el posicionamiento SE0. Las palabras clave -0 keywords- son las expresiones, palabras o frases, que un usuario introduce en un buscador para localizar determinada información. Partiendo de un primer site, Semrush localiza otros sites que utilizan palabras clave idénticas o muy similares y analiza su posicionamiento SE0, ofreciendo así un mapa de competidores muy detallado, ajustado a las búsquedas de los usuarios. Dicho mapa incorpora sites de diferente naturaleza pero afines: gestores de reservas, comparadores de precios, o incluso páginas basadas en la economía colaborativa, donde el intercambio se produce exclusivamente entre particulares.

Una vez identificados los principales sites del sector, se ha realizado una comparativa de las métricas digitales más significativas. Las variaciones en los valores de Alexa y Semrush se deben al uso de algoritmos y fuentes de datos de tráfico diferentes.

Atendiendo a los rankings internacionales de Alexa y Semrush, el site de referencia y líder absoluto del sector es el portal de reservas Booking.com; seguido de Tripadvisor.com. Mucho más retrasados se encuentran Airbnb.com y Kayak.com. De manera global, estos cuatro sites reciben mensualmente 119,5 millones de visitantes y 57,1 millones de visitas únicas, es decir, eliminadas las repeticiones (ver Tabla 1). 
Influencia social online: nuevas tecnologías en la comunicación del sector turístico | 146

MONOGRÁFICO

\begin{tabular}{|c|c|c|c|c|}
\hline Site & Ranking Alexa & Ranking Semrush & $\mathbf{N}^{\circ}$ de visitas & $\begin{array}{c}\mathbf{N}^{\circ} \text { de visitas } \\
\text { únicas }\end{array}$ \\
\hline Booking.com & 125 & 176 & $62,5 \mathrm{M}$ & $28,6 \mathrm{M}$ \\
\hline Tripadvisor.com & 275 & 402 & $29,3 \mathrm{M}$ & $16,2 \mathrm{M}$ \\
\hline Airbnb.com & 445 & 731 & $17,9 \mathrm{M}$ & $7,3 \mathrm{M}$ \\
\hline Kayak.com & 924 & 1.517 & $9,8 \mathrm{M}$ & $5 \mathrm{M}$ \\
\hline
\end{tabular}

Tabla 1: Ranking tráfico mundial sites de alojamientos turísticos

Fuente: elaboración propia a partir de Alexa y Semrush, datos mensuales, marzo 2017.

También en España (ver Tabla 2) el site de referencia es Booking.com, seguido de Tripadvisor.es. Sin embargo, el análisis incorpora algunos sites locales.

\begin{tabular}{|c|c|c|c|c|c|c|}
\hline Site & $\begin{array}{c}\text { Ranking } \\
\text { Alexa } \\
\text { (España) }\end{array}$ & $\begin{array}{c}\mathbf{N}^{\circ} \text { de } \\
\text { visitas }\end{array}$ & $\begin{array}{c}\mathbf{N}^{\circ} \text { de } \\
\text { visitas } \\
\text { únicas }\end{array}$ & $\begin{array}{c}\mathbf{N}^{\circ} \text { de } \\
\text { pag./visita }\end{array}$ & $\begin{array}{c}\text { Tiempo } \\
\text { medio } \\
\text { visita }\end{array}$ & $\begin{array}{c}\text { Tasa de } \\
\text { rebote\% }\end{array}$ \\
\hline $\begin{array}{c}\text { Booking. } \\
\text { com }\end{array}$ & 40 & $14,7 \mathrm{M}$ & $6,9 \mathrm{M}$ & 7,65 & $10: 55$ & 27,80 \\
\hline $\begin{array}{c}\text { Tripadvisor. } \\
\text { es }\end{array}$ & 88 & $4,8 \mathrm{M}$ & $3,1 \mathrm{M}$ & 4,24 & $4: 24$ & 51,01 \\
\hline Airbnb.es & 169 & $1,7 \mathrm{M}$ & $824,1 \mathrm{~K}$ & 10,57 & $13: 35$ & 23,8 \\
\hline Trivago.es & 534 & $1,6 \mathrm{M}$ & $1,2 \mathrm{M}$ & 2,97 & $4: 13$ & 48,9 \\
\hline Rumbo.es & 564 & $1,5 \mathrm{M}$ & $1,3 \mathrm{M}$ & 2,81 & $3: 30$ & 57,33 \\
\hline Kayak.es & 471 & $924,9 \mathrm{~K}$ & $649,1 \mathrm{~K}$ & 3,35 & $4: 36$ & 37,09 \\
\hline Expedia.es & 828 & $665 \mathrm{~K}$ & $540,7 \mathrm{~K}$ & 2,98 & $8: 29$ & 56,3 \\
\hline $\begin{array}{c}\text { Centraldere- } \\
\text { servas.com }\end{array}$ & 3.039 & $205,6 \mathrm{~K}$ & $140,5 \mathrm{~K}$ & 4,67 & $5: 55$ & 39,75 \\
\hline
\end{tabular}

Tabla 2: Principales indicadores digitales de los sites de alojamientos turísticos en España

Fuente: elaboración propia a partir de Alexa y Semrush, datos mensuales, marzo 2017.

Booking.com cuenta con más de 14 millones de visitas mensuales y 6,9 millones de visitas únicas (una vez eliminadas las repeticiones). Durante la vista a esta web, los usuarios ven de media 7,65 páginas -navegan por más de 7 pantallas diferentes dentro del site- e invierten 10:55 minutos de media, lo que sugiere un alto nivel 


\section{MONOGRÁFICO}

de interés. Sin embargo en estos dos indicadores, las mejores cifras las consigue Airbnb.es, con el mayor número de páginas vistas del sector -más de 10 páginas en cada visita- y el tiempo de visita más prolongado, 13:35 minutos. Este resultado es consistente con el tipo de usuarios de este site, basado en la economía colaborativa y donde los comentarios de los usuarios son la base de su funcionamiento.

La tasa de rebote es un indicador negativo que mide el porcentaje de visitas en las que el usuario ha abandonado el site en la página de entrada, sin llegar a interactuar con ella. La tasa de rebote es más favorable cuanto menor sea su valor. Entre los sites analizados destaca Airbnb.es, con una tasa de rebote del 23,8\%, la más baja de todas, aunque próxima a la de Booking.com (27,8\%). Considerando como umbral aceptable el 40\% solo Tripadvisor.es, Trivago.es, Rumbo.es y Expedia.es están por encima.

El análisis de las fuentes de tráfico orienta sobre los niveles de fidelidad de los usuarios hacia estos sites, ofreciendo datos dispares. Mientras Airbnb.es y Booking.com poseen niveles de fidelidad muy satisfactorios ya que en torno al 60 $\%$ de sus usuarios proceden de entradas directas, es decir, teclean el nombre directamente o lo tienen guardado en los favoritos del buscador. Otros por el contrario, como Tripadvisor.es y Trivago.es presentan los valores más bajos en este aspecto. Para ellos su principal fuente de tráfico son los buscadores, con porcentajes superiores al 60-70\%, lo que sugiere una estrategia muy activa de compra de palabras clave, optimización del SE0 (search engine optimization) y el SEM (search engine marketing).

El porcentaje de tráfico que proviene de enlaces externos y redes sociales -denominado tráfico de referencias o referral-indica el nivel de actividad y presencia en otros sites. Solo Expedia.es tiene una presencia significativa de tráfico procedente de referencias. En general, los sites analizados presentan niveles bajos y muy bajos como sucede con Kayak.es y Tripadvisor.es (ver Tabla 3).

\begin{tabular}{|c|c|c|c|c|}
\hline Site & Entradas directas & Buscadores & Redes sociales & Enlaces \\
\hline Booking.com & $59,74 \%$ & $27,79 \%$ & $2,44 \%$ & $10,04 \%$ \\
\hline Tripadvisor.es & $15,80 \%$ & $77,76 \%$ & $0,56 \%$ & $5,88 \%$ \\
\hline Airbnb.es & $61,86 \%$ & $26,43 \%$ & $2,10 \%$ & $9,61 \%$ \\
\hline
\end{tabular}




\begin{tabular}{|c|c|c|c|c|}
\hline Site & Entradas directas & Buscadores & Redes sociales & Enlaces \\
\hline Trivago.es & $21,37 \%$ & $61,04 \%$ & $8,62 \%$ & $8,97 \%$ \\
\hline Rumbo.es & $32,25 \%$ & $58,71 \%$ & $0,67 \%$ & $8,37 \%$ \\
\hline Kayak.es & $52,78 \%$ & $41,11 \%$ & $1,44 \%$ & $4,67 \%$ \\
\hline Expedia.es & $43,41 \%$ & $27,80 \%$ & $0,18 \%$ & $28,61 \%$ \\
\hline $\begin{array}{c}\text { Centraldereser- } \\
\text { vas.com }\end{array}$ & $30,43 \%$ & $61,35 \%$ & $0,00 \%$ & $8,21 \%$ \\
\hline
\end{tabular}

* Orden de los sites según Tabla 2

Tabla 3: Fuentes de tráfico

Fuente: elaboración propia a partir de datos de Alexa, marzo 2017.

Junto a las diferencias encontradas en el tráfico, su procedencia, el tiempo de navegación, el grado de interés o la fidelidad de los usuarios, también se han identificado discrepancias interesantes en los mecanismos de influencia que emplean cada uno de los sites analizados. Todas las ofertas de alojamiento incluyen información de interés de diversa naturaleza (Imagen 1), como el precio, la ubicación o el número de habitaciones disponibles pero además, se presentan datos numéricos a los que se da visibilidad y presencia: la evaluación numérica de la oferta y número de comentarios que ha recibido. Esta información puede proporcionarse de diferente forma. La Tabla 4 detalla las características de cada uno y sus peculiaridades.

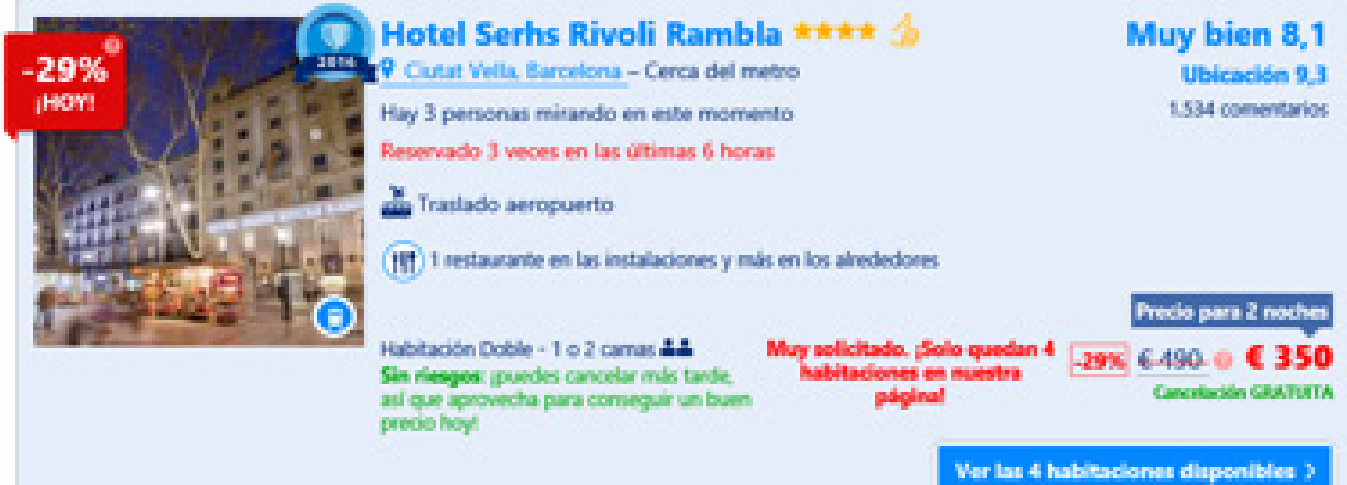

Figura 1: Contenidos informativos en una oferta hotelera Fuente: $h t t p: / / w w w . b o o k i n g . c o m$ 


\section{MONOGRÁFICO}

\begin{tabular}{|c|c|c|c|}
\hline Site & Actividad & Comentarios & $\begin{array}{c}\text { Información } \\
\text { (mecanismos de } \\
\text { influencia) }\end{array}$ \\
\hline Booking.com & Gestor reservas & Propios clientes & $\begin{array}{l}\text { Evaluación numérica } \\
\text { (sobre } 10 \text { ) } \\
\text { Equivalencia en texto } \\
\text { (sobre } 5, \text { de fantásti- } \\
\text { co a muy mal) } \\
\text { Volumen de } \\
\text { comentarios }\end{array}$ \\
\hline Tripadvisor.es & $\begin{array}{l}\text { Gestor reservas y } \\
\text { comparador }\end{array}$ & Propios clientes & $\begin{array}{c}\text { Icono puntuación } \\
\text { media (sobre 5) } \\
\text { Puesto en el ranking } \\
\text { Volumen de } \\
\text { comentarios } \\
\text { Gráfico distribución } \\
\text { comentarios (sobre } \\
\text { 5, de excelente a } \\
\text { pésimo) }\end{array}$ \\
\hline Airbnb.es & $\begin{array}{c}\text { Gestor reservas entre } \\
\text { particulares }\end{array}$ & Propios clientes & $\begin{array}{c}\text { Icono puntuación } \\
\text { media (sobre 5) } \\
\text { Volumen de } \\
\text { comentarios } \\
\end{array}$ \\
\hline Trivago.es & $\begin{array}{l}\text { Gestor reservas y } \\
\text { comparador }\end{array}$ & $\begin{array}{l}\text { Clientes de los sites de } \\
\text { la oferta de referencia }\end{array}$ & $\begin{array}{c}\text { Puesto en el ranking } \\
\text { Trivago Index (sobre } \\
\text { 100) } \\
\text { Volumen de } \\
\text { comentarios } \\
\text { Icono puntuación } \\
\text { media (sobre 5) } \\
\text { Equivalencia en texto } \\
\text { (sobre 5, de excelente } \\
\text { a regular) }\end{array}$ \\
\hline Rumbo.es & Gestor reservas & Sistema trustyou & $\begin{array}{c}\text { Evaluación numérica } \\
\text { (sobre 100) } \\
\text { Equivalencia en texto } \\
\text { Volumen de } \\
\text { comentarios } \\
\text { Icono puntuación } \\
\text { media (sobre 5) }\end{array}$ \\
\hline
\end{tabular}


MONOGRÁFICO

\begin{tabular}{|c|c|c|c|}
\hline Site & Actividad & Comentarios & $\begin{array}{c}\text { Información } \\
\text { (mecanismos de } \\
\text { influencia) }\end{array}$ \\
\hline Kayak.es & $\begin{array}{c}\text { Gestor reservas y } \\
\text { comparador }\end{array}$ & $\begin{array}{l}\text { Clientes de los sites de } \\
\text { la oferta de referencia }\end{array}$ & $\begin{array}{l}\text { Evaluación numérica } \\
\text { (sobre } 10 \text { ) } \\
\text { Icono puntuación } \\
\text { media (sobre } 5 \text { ) } \\
\text { Equivalencia en texto } \\
\text { (sobre } 4 \text {, de excelente } \\
\text { a regular) } \\
\text { Volumen de } \\
\text { comentarios }\end{array}$ \\
\hline Expedia.es & Gestor reservas & Propios clientes & $\begin{array}{l}\text { Evaluación numérica } \\
\text { (sobre 10) } \\
\text { Equivalencia en texto } \\
\text { Volumen de comenta- } \\
\text { rios propios } \\
\text { \% de recomendación } \\
\text { Icono cara } \\
\text { Clasificación en } \\
\text { Tripadvisor }\end{array}$ \\
\hline Centraldereservas.com & Gestor reservas & Propios clientes & $\begin{array}{c}\text { Evaluación numérica } \\
\text { (sobre 10) } \\
\text { Icono puntuación } \\
\text { media (sobre 5) } \\
\text { Volumen de } \\
\text { comentarios }\end{array}$ \\
\hline
\end{tabular}

Tabla 4: Comparativa mecanismos de influencia en los sites de alojamientos turísticos en España Fuente: elaboración propia.

\section{Material y métodos}

La importancia de la influencia social online en el marco de los sites de alojamientos, nos conduce al objetivo principal del presente estudio. Este trabajo propone analizar la influencia social que ejercen las evaluaciones numéricas y el volumen de comentarios en el proceso de decisión de los usuarios de los sites de alojamientos turísticos, teniendo en cuenta el papel que desempeña la susceptibilidad del propio usuario a la información que procede de otras personas. 


\section{MONOGRÁFICO}

A partir de este objetivo principal, se plantean los siguientes objetivos secundarios relativos a los posibles efectos de los factores de influencia en la conducta de compra del usuario de los sites de alojamientos:

- Estudiar el efecto en la atención. Se trata de analizar cómo las variables de estudio: evaluaciones numéricas, volumen de comentarios y susceptibilidad a la influencia, afectan a la atención que suscita una oferta de alojamiento. Se desea conocer en qué medida una oferta llama más la atención cuando posee una evaluación alta frente a baja, o cuando está acompañada de muchos o pocos comentarios. Asimismo, la propia personalidad del usuario, más o menos sensible a la influencia social, podría afectar a la atención que despierta la oferta.

- Estudiar el efecto en la estimación de la capacidad informativa de una oferta. El objetivo es analizar cómo las variables de estudio -evaluaciones numéricas, volumen de comentarios y susceptibilidad a la influencia- pueden alterar la estimación que hace el usuario sobre el contenido informativo de una oferta.

- Estudiar el efecto en el interés que despierta una oferta para ser preseleccionada. Se desea medir la influencia de las evaluaciones y el volumen de comentarios en la consideración de una oferta como posible alternativa. Se contempla que la susceptibilidad del usuario a la influencia social modere este efecto.

- Estudiar el efecto en la probabilidad de selección de una oferta. En este caso, más allá del interés, se analiza la probabilidad de seleccionar definitivamente una oferta a partir de la información que recibe con la evaluación numérica y el volumen de comentarios.

Para dar respuesta a estos objetivos se ha optado por un enfoque empírico realizando un experimento. La elección de esta técnica se justifica por los objetivos del estudio donde se plantean relaciones de causalidad entre las evaluaciones numéricas y el volumen de comentarios, que adoptan el papel de variables independien- 
tes y los presuntos efectos sobre atención, información, interés y probabilidad de reservar una oferta de alojamiento, en adelante variables dependientes. La experimentación está considerada como una de las técnicas más adecuadas para verificar relaciones de causalidad (Hair, Anderson, Tatham y Black, 1999).

Se realiza por lo tanto un experimento con un diseño factorial donde se comparan los efectos de 2 niveles de evaluación numérica (alta $v s$ baja) y 3 niveles de comentarios (muchos-pocos-ninguno), sobre las variables dependientes (atención, información, interés y probabilidad de reserva), controlando el efecto de la susceptibilidad del sujeto a la influencia social.

\section{Procedimiento}

La recolección de datos para el experimento se realizó empleando un formulario de preguntas cerradas en escalas. Antes de responder, se informaba a los participantes de que se trataba de un estudio académico sobre los sites de alojamientos sin aportar más detalles. Los participantes debían responder a una batería de preguntas con respuestas en escala Likert-5 y Likert-7 sobre tres áreas temáticas:

- Área temática 1: Actividad del usuario en la red.

- Área temática 2: Uso de las webs de reserva para contratar hoteles.

- Área temática 3: Susceptibilidad del usuario a la influencia social.

El cuestionario comenzaba en el área temática 1 con preguntas sencillas sobre el uso de las redes sociales. Al inicio del área temática 2 se planteaba al encuestado un escenario imaginario de viaje con detalles sobre duración, compañía, destino, etc., para el que debía realizar una reserva en un site de alojamiento, en cuya búsqueda encontraba la oferta estímulo del experimento. A continuación se le presentaban las preguntas sobre dicha oferta. Posteriormente el encuestado continuaba con el área temática 3 donde se recogían datos sobre su susceptibilidad a la influencia social y terminaba con los datos de clasificación y el agradecimiento por la colaboración. 


\section{MONOGRÁFICO}

El 10,5\% de los participantes fueron contactados posteriormente para preguntarles sobre el objetivo del cuestionario de forma abierta. Ninguno mencionó el posible carácter experimental del mismo.

\section{Diseño de los estímulos}

Se diseñaron 6 modelos de cuestionarios, en cada uno de los cuales se empleaba una oferta estímulo diferente con distinta evaluación numérica del hotel y un volumen mayor o menor de comentarios, conforme al diseño que se adjunta en la Tabla 5:

\begin{tabular}{|c|c|c|}
\hline Oferta 1 & \multirow{4}{*}{ Evaluación numérica alta } & Muchos comentarios \\
Oferta 2 & & Pocos comentarios \\
\hline Oferta 3 & \multirow{4}{*}{ Evaluación numérica baja } & Ningún comentario \\
\hline Oferta 4 & & Muchos comentarios \\
\hline Oferta 5 & & Pocos comentarios \\
\hline Oferta 6 & & Ningún comentario \\
\hline
\end{tabular}

Tabla 5: Diseños de estímulos experimentales

Para evitar la influencia de otros posibles elementos dentro de la oferta se optaron por las siguientes medidas:

- Precio: se omitía el precio. En su lugar figuraba un icono de "Mejor precio".

- Imagen en la oferta: como imagen del hotel se seleccionó una foto detalle de un cartel de hotel, de baja capacidad diagnóstica, de modo que los usuarios apenas pudiera extraer información de la imagen. Se utilizó la misma imagen en todas las ofertas.

Para asegurar la validez del diseño de las ofertas se realizó una reunión de grupo con usuarios de los sites de alojamientos donde, entre otros temas, se abordó el debate sobre lo que se consideraba una evaluación numérica alta y baja y sobre el volumen de comentarios que se interpretaban como muchos o pocos, que sirvieron de valores de referencia en el diseño de los estímulos experimentales. 
A partir de esta información se diseñaron las ofertas (Imagen 2) que se sometieron a la evaluación de una muestra de 60 usuarios potenciales. Estos sujetos respondieron a preguntas sobre la similitud entre el diseño realizado y las ofertas que se presentan en los sites de alojamientos, así como sobre la claridad de la información contenida en la oferta. Empleando como instrumento de medida una escala de 7 posiciones ambos datos fueron satisfactorios. La similitud de las ofertas diseñadas obtuvo una media de 6,05 (DS $=1,13$ ) y la claridad informativa también estaba por encima de $5(M=5,68 ; D S=1,17)$. El diseño se asemejaba a una oferta para el $84 \%$ de la muestra y era comprensible para el $89 \%$.

Los 6 modelos de cuestionarios se programaron en la plataforma de Google Forms.
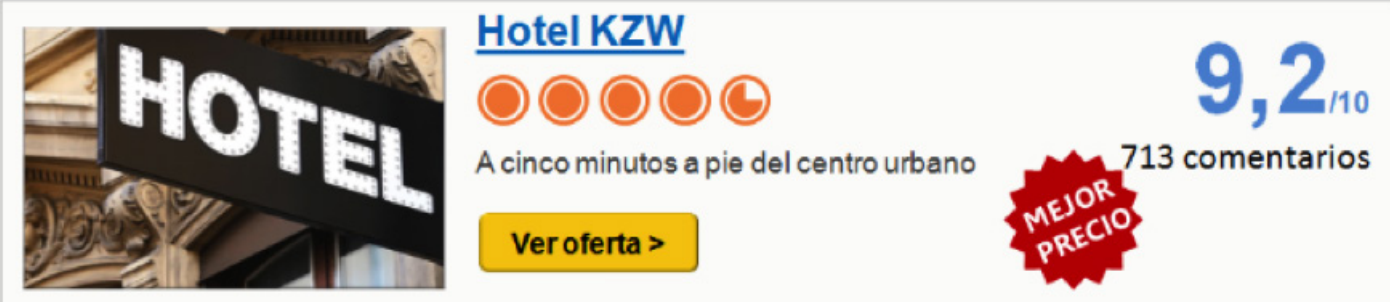

Figura 2: Oferta 1. Evaluación numérica alta con muchos comentarios

\section{Muestra}

Para la recogida de datos se contó con una muestra formada por 213 sujetos seleccionados por conveniencia que atendían al criterio genérico de ser usuarios de internet más o menos activos y específicamente en la contratación de viajes, con edades comprendidas entre los 27 y 53 años. Cada participante fue asignado por azar a una de las 6 ofertas (celdas). El tamaño final de las celdas oscila entre $31 \mathrm{y}$ 47 participantes.

En la composición de la muestra encontramos un 53\% de mujeres y un $47 \%$ de hombres, con edades comprendidas entre los 27 y los 53 años. Si bien el intervalo de edad es amplio, la muestra es bastante homogénea en la conducta observada, donde los usuarios manifiestan un elevado nivel de experiencia. 


\section{MONOGRÁFICO}

Todos los participantes están activos en alguna red social (100\%) y muchos $(63,2 \%)$ lo están en más de una red social. Más del $50 \%$ viaja de 4 a 12 veces al año. El 50\% tiene una probabilidad de 0,7 o superior de alojarse en un hotel. La probabilidad de reservar el hotel por internet es del $90 \%$ para el $50 \%$ de la muestra.

El análisis de las respuestas dadas por los sujetos a la actividad que realizan en la red nos sitúa frente a dos tipos de usuarios que etiquetamos como usuarios intensos y usuarios activos. En ambos casos se trata de personas acostumbradas a utilizar habitualmente internet, aunque la diferencia estriba en el grado de intensidad. El usuario intenso (57,3\%) se informa, contacta con amigos y accede a contenidos a diario a través de internet; mientras que el usuario activo $(42,7 \%)$ se informa a diario través de internet pero es menos participativo en redes sociales y presenta tasas más bajas de consumo de contenidos y contratación de ofertas.

\begin{tabular}{|c|c|c|c|}
\hline Utilizo internet para... & $\begin{array}{c}\text { Usuarios } \\
\text { intensos } \\
\mathbf{5 7 , 3} \%\end{array}$ & $\begin{array}{c}\text { Usuarios } \\
\text { activos } \\
\mathbf{4 2 , 7} \%\end{array}$ & Sig. \\
\hline Leer noticias (diarios/revistas) & 3,52 & 3,04 &, 000 \\
\hline Realizar operaciones bancarias & 2,43 & 2,35 &, 462 \\
\hline Mantener contacto con amigos & 3,66 & 2,49 &, 000 \\
\hline Escuchar música/ver películas & 3,40 & 1,89 &, 000 \\
\hline Participar y compartir (leer/dar opiniones) & 2,97 & 1,85 &, 000 \\
\hline Comprar productos o contratar servicios & 2,42 & 1,99 &, 000 \\
\hline Buscar información antes de decidirme a comprar & 2,89 & 2,21 &, 000 \\
\hline Encontrar ideas/información sobre mis aficiones & 3,16 & 2,27 &, 000 \\
\hline
\end{tabular}

Tabla 6: Tipología de usuarios en la muestra

\section{Escalas de medida}

Se emplearon 4 escalas para la medición del efecto de los modelos de oferta, $\mathrm{y}$ una escala para controlar la susceptibilidad del usuario a la influencia social, que se describen a continuación:

Atención: Capacidad de la oferta presentada para llamar la atención del usuario. Constaba de 4 ítems, valorados en una escala Likert-7, con un alfa de Cronbach de ,725. 
Influencia social online: nuevas tecnologías en la comunicación del sector turístico | 156

- Me llama la atención

- Haría click en la opción para saber más del hotel

- Me he fijado en la puntuación del hotel

- Me he fijado en el número de comentarios

Información: Capacidad informativa de la oferta presentada. Constaba de 4 ítems valorados en una escala Likert-7 con un alfa de Cronbach de ,905.

- Útil para ayudarme a decidir

- Suficiente para tenerla en cuenta

- Informativa

- Confiable

Intención: Interés que despierta la oferta presentada como opción de compra, empleando para ello 3 ítems valorados en una escala Likert-7 con un alfa de Cronbach de ,956.

- Me interesaría por este hotel

- Incluiría este hotel como una alternativa posible

- Creo que esta sería una buena alternativa

Probabilidad de reserva: pregunta numérica sobre la probabilidad de que el usuario reservara en ese hotel en escala de 0 a 10.

Susceptibilidad del usuario a la influencia social. El interés por controlar la posible influencia que ejerce esta variable en las decisiones de los usuarios a la hora 


\section{MONOGRÁFICO}

de realizar una reserva justifica su inclusión en el estudio como covariable, medida con 4 ítems de la escala de Bearden et al. (1989) valorados en una escala Likert-7 con un alfa de Cronbach de ,859.

- Cuando compro, a menudo consulto con otras personas

- Para estar seguro de lo que compro me fijo en lo que compran y usan los demás

- Si conozco poco un producto, suelo preguntar a familiares / amigos / conocidos si ellos lo conocen

- Antes de comprar algo suelo pedir la opinión de otras personas

Una vez realizado el análisis de fiabilidad de las escalas y verificada su calidad se agregaron los ítems calculando el valor medio para cada variable.

A continuación se estimaron los valores del modelo de covarianza donde las variables independientes fueron: evaluación numérica y número de comentarios; las variables dependientes: atención, información, interés y probabilidad de reserva, y como covariable se incluyó la susceptibilidad del usuario a la influencia social. Para el análisis estadístico se empleó el programa SPSS versión 21.0.

\section{Resultados}

La exposición de los resultados se organiza del siguiente modo: se presenta primero la influencia de la evaluación numérica, seguida del efecto del volumen de comentarios, después el efecto conjunto de ambos factores y por último el papel de la susceptibilidad.

\section{La influencia de la evaluación numérica}

Se comparan las respuestas de los participantes cuando se enfrentaron a una oferta con una evaluación alta frente a una oferta con una evaluación baja. Tras verificar que los datos satisfacen el test de Levene, se obtiene que las 
Influencia social online: nuevas tecnologías en la comunicación del sector turístico | 158

evaluacionesnuméricasinfluyensignificativamenteenlaatención que suscitala oferta $F(1,206)=27,8, p<, 000$, en la intención $F(1,206)=116,19, p<, 000 \mathrm{y}$ en la probabilidad de reservarla $F(1,206)=110,51, p<, 000$.

Una evaluación numérica alta llama más la atención que una baja (Gráfico 1), y provoca una intención de reserva significativamente mayor (Gráfico 2). No obstante, siendo ambas variables significativas, el efecto en la atención es menos intenso que en la intención de reserva.

Interesa señalar que la evaluación numérica alta o baja no afecta a la estimación de la capacidad informativa de una oferta.

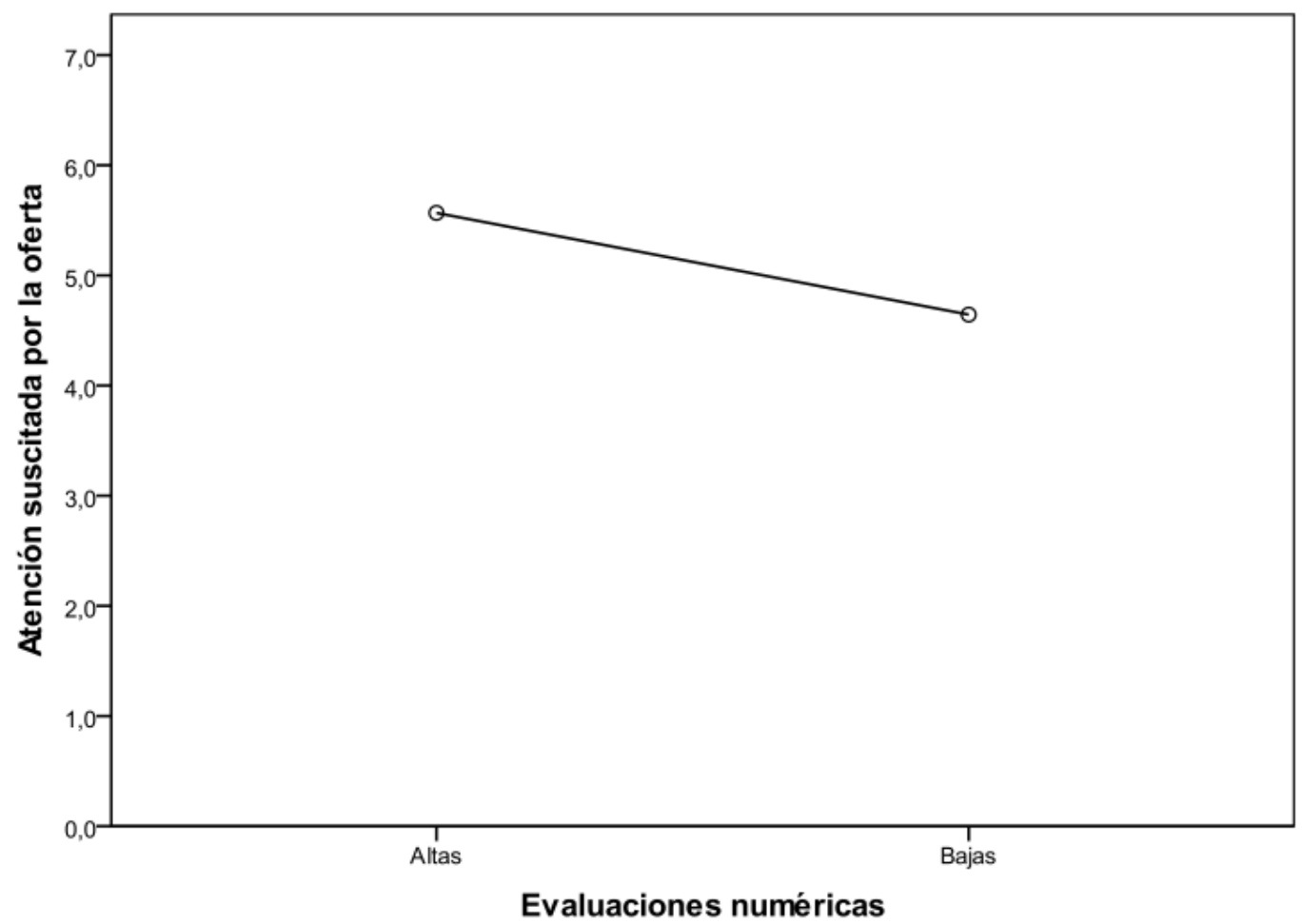

Las covariables que aparecen en el modelo se evalúan en los siguiente valores: SSII = 4,7101

Gráfico 1: Valores medios de la atención para ofertas con evaluaciones altas frente a bajas. 


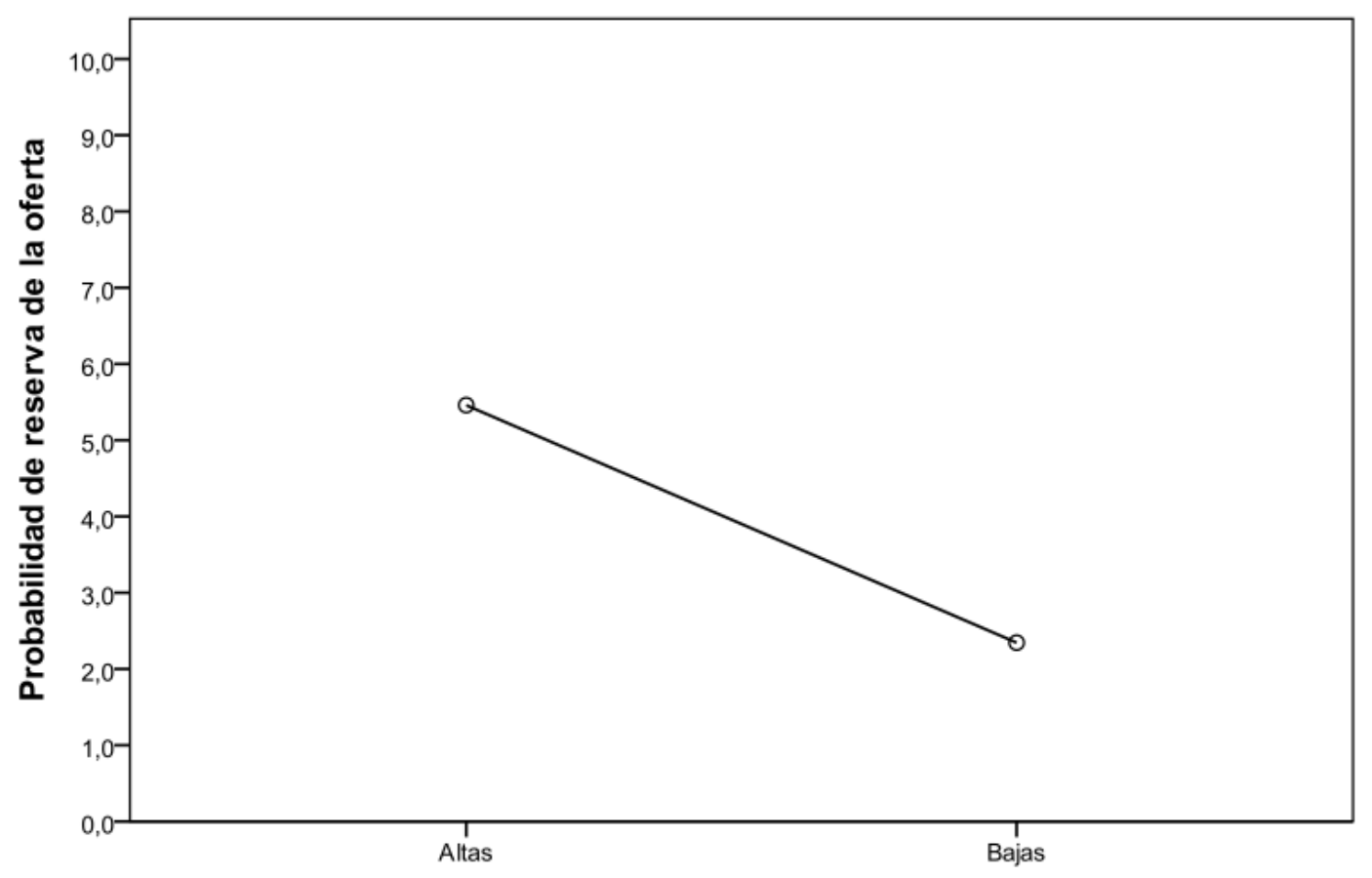

Evaluaciones numéricas

Las covariables que aparecen en el modelo se evalúan en los siguiente valores: SSII = 4,7101

Gráfico 2: Valores medios de la probabilidad de reserva para ofertas con evaluaciones altas frente a bajas.

\section{La influencia del volumen de comentarios}

A diferencia de lo que sucedía con la evaluación numérica, el volumen de comentarios solo influye significativamente en la estimación de la capacidad informativa de una oferta $F(2,206)=7,84, p<, 00$. Como muestra el Gráfico 3, conforme se reduce el volumen de comentarios el usuario considera que la oferta es menos informativa y confiable. En otras palabras, tener muchos comentarios, aunque el usuario no los haya leído, crea una buena expectativa de información referida a la oferta. 
Influencia social online: nuevas tecnologías en la comunicación del sector turístico | 160

MONOGRÁFICO

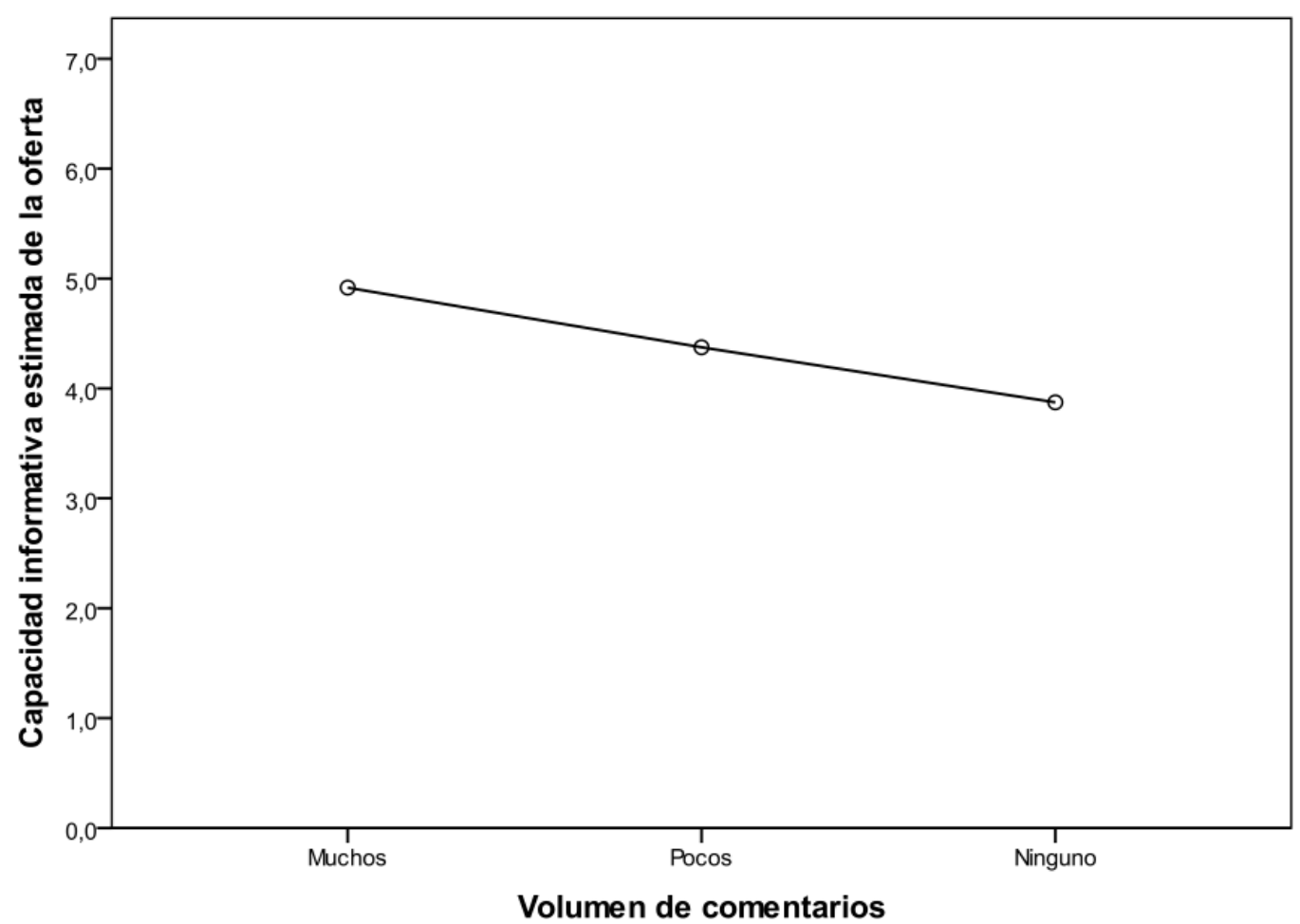

Las covariables que aparecen en el modelo se evalúan en los siguiente valores: SSII = 4,7101

Gráfico 3: Valores medios de la capacidad informativa de una oferta para ofertas con muchos, pocos o ningún comentario.

Ahora bien, el volumen de comentarios no influye significativamente en la atención que suscita la oferta en el usuario, es decir, tener muchos o pocos comentarios ni incrementa ni reduce la atención que despierta una oferta en el potencial viajero.

El volumen de comentarios aumenta la probabilidad de reservar una oferta $F$ $(2,206)=3,7, p<, 05$. Esto significa que el volumen de comentarios puede actuar como un reductor del riesgo de decisión. Ante ofertas acompañadas de muchos comentarios, el usuario considera que la oferta es más informativa y por ello anticipa que decidirá mejor, con menos riesgo, lo que se incrementa su probabilidad final de elección. 


\section{MONOGRÁFICO}

\section{La influencia de las evaluaciones numéricas y el volumen de comentarios}

Cuando se comparan los resultados de las 6 alternativas creadas que combinan evaluaciones numéricas altas y bajas con diferente volumen de comentarios (muchos, pocos o ninguno) el efecto conjunto es significativo en la intención $F(2,206)$ $=4,64, p<, 05 \mathrm{y}$ en la probabilidad de reservar una oferta $F(2,206)=4,83, p<, 05$.

Las ofertas con evaluaciones altas son siempre más atractivas que las ofertas con evaluaciones bajas, pero es notorio que un bajo número de comentarios hace decaer el interés de una oferta, sin haber leído los comentarios. Este efecto es significativo en el caso de las evaluaciones altas pero no para las evaluaciones bajas, donde tener muchos o pocos comentarios no es capaz de cambiar el efecto de una evaluación baja (Gráfico 4).

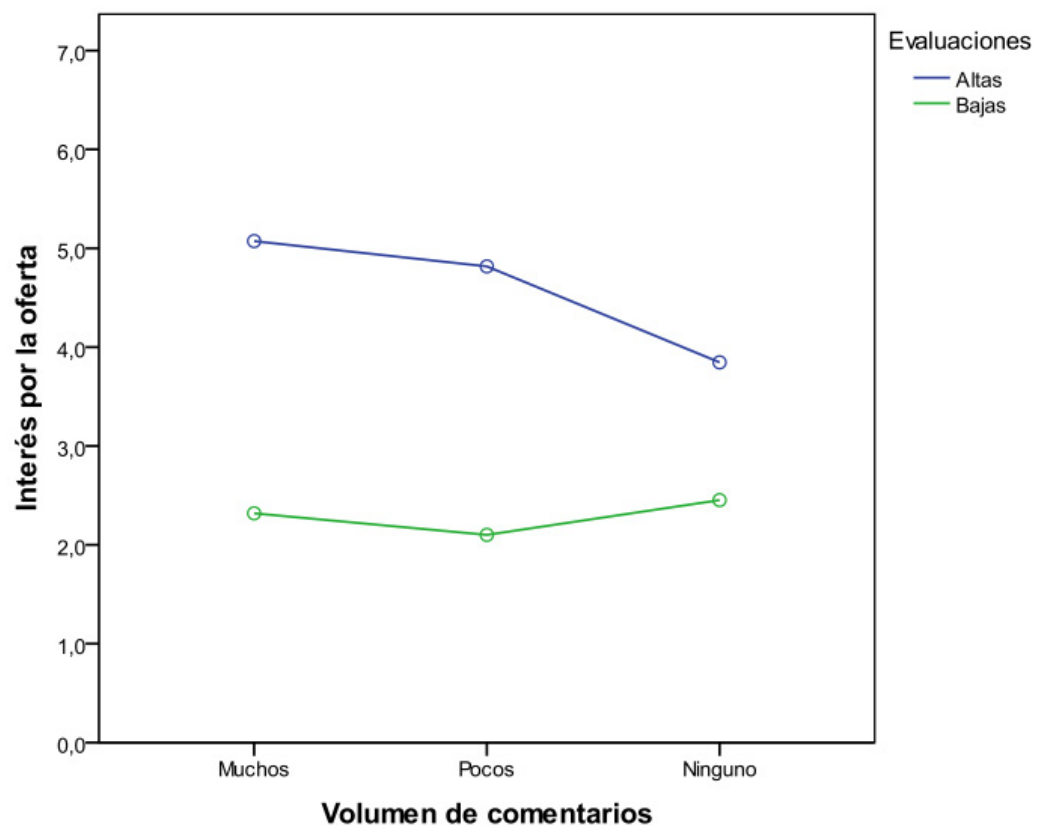

Las covariables que aparecen en el modelo se evalúan en los siguiente valores: $S S \|=4,7101$

Gráfico 4: Valores medios de la intención de reserva por diferente volumen de comentarios. 


\section{El papel de la susceptibilidad del usuario a la influencia de las evaluaciones numéricas y al volumen de comentarios}

Se verifica que no hay diferencias en los grupos respecto a la susceptibilidad de los participantes a la influencia social, de este modo nos aseguramos de que los grupos son aleatorios en cuanto a su composición, por lo que en todos hay usuarios con diferentes niveles de susceptibilidad.

La susceptibilidad influye de forma significativa en que los sujetos se fijen en ciertas ofertas $F(1,206)=4,64 p<, 05 \mathrm{y}$ en que las consideren informativas $F(1,206)=6,07 p<, 05$ pero no en el interés, ni en sus probables decisiones de compra.

Esto significa que la susceptibilidad es un rasgo de personalidad que aumenta la sensibilidad del usuario a lo que dicen otros usuarios sobre las opciones de consumo. Estos usuarios con elevada susceptibilidad prestan más atención a la evaluación y extraen más información del hecho de que una opción sea comentada por otras personas. Ahora bien, cuando satisfacen su necesidad de información, el patrón de selección que adoptan es similar al de los usuarios menos susceptibles.

\section{Discusión}

El objetivo de este trabajo era estudiar la influencia de las evaluaciones numéricas y el volumen de comentarios en las ofertas de los sites de alojamientos sobre diferentes aspectos del proceso de decisión de los usuarios: atención, estimación de la capacidad informativa de una oferta, interés y probabilidad de reserva. Los resultados indican que la evaluación numérica tiene un papel esencial en el proceso de decisión, y su efecto es significativamente mejor si además se acompaña de muchos comentarios. Por el contrario, una evaluación baja, tanto si se acompaña de muchos como de pocos comentarios, es una opción poco atractiva que el usuario tenderá a descartar.

La incorporación en el modelo de un rasgo de personalidad como es la susceptibilidad del usuario aporta además un valor añadido al estudio al poner en evidencia 
que la influencia de evaluaciones y comentarios está moderada por la forma de ser del propio usuario. Los consumidores más sensibles se sentirán más cómodos ante ofertas evaluadas positivamente por muchas personas.

Un aspecto a destacar en el estudio realizado es el hecho de que toda la influencia analizada se produce en la primera etapa de este proceso de elección de alojamiento turístico, cuando el objetivo es reducir el universo de alternativas a un conjunto numéricamente manejable de opciones altamente relevantes. Los usuarios buscan información rápida de evaluar y confiable. Los tiempos medios de visita de estos sites corroboran este hecho.

La evaluación numérica es un indicador fácil de comprender que satisface la necesidad de rapidez. Por su parte, los comentarios otorgan confianza y capacidad informativa a las ofertas cuando el volumen de los mismos es elevado; se trata de información respaldada por la prueba social que es como se conoce al uso de la conducta de otras muchas personas como patrón de referencia en una decisión.

El presente estudio confirma el papel relevante que ha adquirido el entorno virtual para el consumidor a la hora de buscar información acerca de un producto o servicio facilitando y simplificando su elección de compra como ya indicaban estudios previos (Shim, Eastlick, Lotz, y Warrington, 2001).

Como reflejan los resultados obtenidos, la información que generan y proporcionan otros usuarios con sus valoraciones, comentarios y experiencias ejerce una influencia social que resulta creíble, útil y precisa para los consumidores cuando se encuentran ante una elección de alojamiento turístico. Se constata que esta influencia social puede ser ejercida mediante tres mecanismos, evaluaciones numéricas, comentarios y recomendaciones. Tras el análisis de los resultados, queda patente que evaluaciones numéricas y comentarios hechos por usuarios anónimos a través de las plataformas tecnológicas reducen el riesgo, facilitan la toma de decisiones y permiten la interacción entre quienes comparten intereses, confirmando así planteamientos de otros estudios (Amblee y Bui, 2011). 
En línea con el informe Nielsen (2012) comentado en el marco teórico, se confirma que la información expresada en forma de números efectivamente ayuda a cribar alternativas. Los números son considerados por tanto, información solvente para el consumidor de alojamientos turísticos. En este sentido, las empresas de alojamientos turísticos deben tener en consideración este fenómeno de influencia social online y los retos que esto les plantea tal y como señalaban Litvin, Goldsmith y Pan (2008). Conocer estos mecanismos de influencia resulta relevante para que las empresas del sector turístico puedan captar, analizar, interpretar y gestionar la participación de los usuarios de forma que puedan obtener ventajas competitivas, dada la importancia y repercusión que tienen en las decisiones de los consumidores.

El estudio realizado ofrece además conclusiones importantes para las empresas del sector turístico y en general, para todas aquellas comunidades que explotan la influencia social (cine, compras...). En primer lugar, destacar la importancia de las evaluaciones numéricas presentadas como números claramente visibles. Su presencia aumenta el atractivo de las ofertas, especialmente para las personas más susceptibles a la influencia social, proporcionando una información rápida y sencilla. Sin embargo, no todos los sites las emplean. En algunos casos como Tripadvisor este dato se sustituye por un icono, la posición en el ranking, las frecuencias de las puntuaciones, pero no se presenta el dato claro del número, lo que tal vez reduce la claridad, dificulta la comprensión y la comparación entre las ofertas.

En segundo lugar, alertar a los alojamientos de la conveniencia de estimular a sus huéspedes para que comenten su estancia. Tal y como se ha observado, aún con una evaluación alta, tener pocos comentarios puede ser peligroso.

Por último, llamar la atención sobre el hecho de que la influencia social que se ejerce a través de los mecanismos analizados, no reside sólo en el dato sino en las características personales de quienes observa esos datos. Esto es así, como también lo es el hecho de que vivimos constantemente estimulados por las opiniones de los otros, a las que damos cada vez más importancia y credibilidad. 


\section{MONOGRÁFICO}

\section{Referencias}

Amblee, N. \& Bui, T. (2011). Harnessing the influence of social proof in online shopping: The effect of electronic word of mouth on sales of digital microproducts. International Journal of Electronic Commerce, 16(2), 91-114. doi: 10.2753/JEC1086-4415160205

Anderson, E.W. (1998). Customer satisfaction and word of mouth. Journal of Service Research, 1(1), 5-17. doi: 10.1177/109467059800100102

Bearden, W.0., Netemeyer, R.G. \& Teel, J.E. (1989). Measurement of Consumer Susceptibility to Interpersonal Influence. Journal of Consumer Research, 15(4), 473-481. doi: 10.1086/209186

BrightLocal (2014). Local Consumer Review Survey. (https://goo.gl/sywFgy) (2017-2-8).

Burnkrant, R.E. \& Cousineau, A. (1975). Informational and normative social influence in buyer behavior. Journal of Consumer research, 2(3), 206-215. doi: $10.1086 / 208633$

Chevalier, J. \& Mayzlin, D. (2006). The Effect of Word of Mouth on Sales: Online Book Reviews. Journal of Marketing Research, 43(3), 345-354. doi: 10.1509/ jmkr.43.3.345

Cialdini, R. (2009). Influence: Science and Practice. Boston, MA: Pearson Education. ComScore (2007). Online Consumer-Generated Reviews Have Significant Impact on Offline Purchase Behavior. (https://goo.gl/XC21h8) (2017-1-28).

Dellarocas, C. \& Narayan, R. (2006). A statistical measure of a population's propensity to engage in post-purchase online word-of-mouth. Statistical Science, 21(2), 277-285. doi: 10.1214/088342306000000169

Diana-Jens, P. \& Rodríguez, A. (2015). La reputación online y su impacto en la política de precios de los hoteles. Cuadernos de Turismo, 36, 29-155. doi:10.6018/turismo.36.230911

Dichter, E. (1966). How word-of-mouth advertising works. Harvard Business Review, 44(6), 147-160.

Duan, W., Gu, B. \& Whinston, A.B. (2008). The dynamics of online word-of-mouth and product sales-An empirical investigation of the movie industry. Journal of retailing, 84(2), 233-242. doi: 10.1016/j.jretai.2008.04.005 
Influencia social online: nuevas tecnologías en la comunicación del sector turístico | 166

MONOGRÁFICO

Foro Económico Mundial (2015). The Travel \& Tourism Competitiveness Report 2015. (https://goo.gl/z9mnHE) (2017-2-16).

Gutiérrez-Taño, D., Bulchand, J., Díaz, R.J., \& Parra, E. (2013). Antecedentes del uso de los medios sociales por el turista: motivación, oportunidad y capacidad. Cuadernos de Turismo, 31, 153-173. (https://goo.gl/wSP6xK) (2017-2-11).

Hair, J.F. Jr., Anderson, R. E., Tatham, R. L. \& Black, W. C. (1999). Análisis multivariante (5a Ed.). Nueva York: Prentice Hall.

Lee, H., Law, R. \& Murphy, J. (2011). Helpful Reviewers in TripAdvisor, an Online Travel Community. Journal of Travel \& Tourism Marketing, 28(7). doi: 10.1080/10548408.2011.611739

Lee, M. Shi, N. Cheung, C., K Lim, K. \& Sia, C. (2011). Consumer's Decision to Shop Online: The Moderating Role of Positive Informational Social Influence. Information \& Management, 48(6), 185-191. doi: 10.1016/j.im.2010.08.005

Litvin, S.W., Goldsmith, R. \& Pan, B. (2008). Electronic word-of-mouth in hospitality and tourism management. Tourism Management, 29, 458-468. doi: 10.1016/j. tourman.2007.05.011

Martínez-María, M.D., Bernal, J. \& Mellinas, J. (2012). Los hoteles de la región de Murcia ante las redes sociales y la reputación online. Revista de Análisis Turístico, 12(1), 1-10. (https://goo.gl/F7o9BA) (2017-06-13).

Moe, W. \& Schweidel, D. (2011). Online Product Opinions: Incidence, Evaluation and Evolution. Marketing Science, 31(3), 372-386. doi: 10.1287/mksc.1110.0662

Nielsen (2012). State of the Media: The Social Media Report. (https://goo.gl/ ucsh8r) (2016-12-20).

Ögüt, H. \& Onur, B.K. (2012). The influence of internet customer reviews on the online sales and prices in hotel industry. The Service Industries Journal, 32(2). doi: 10.1080/02642069.2010.529436

Olabarri, M.E., Monge, S. \& Usín, S. (2015). Cómo evalúan los jóvenes las opiniones de otros consumidores en Internet. El caso de la UPV/EHU. Revista Latina de Comunicación Social, 70, 703-725. doi: 10.4185/RLCS-2015-1067

Parra, E., Bulchandl, J., Gutiérrez, D. \& Díaz, R. (2010). Intentions to use social media in organizing and taking vacation trips. Computers in Human Behavior, 27(2), 640-654. doi: 10.1016/j.chb.2010.05.022

Peterson, R. A. \& Merino, M. C. (2003). Consumer information search behavior and the Internet. Psychology and Marketing, 29(2), 99-121. doi: 10.1002/mar.10062 
PhoCusWright (2013). Travel Tech Trends. (https://goo.gl/eoWW0j) (2016-12-21) Rao, H., Greve, H.R. \& Davis, G.F. (2001). Fool's gold: Social proof in the initiation and abandonment of coverage by Wall Street analysts. Administrative Science Quarterly, 46(3), 502-526. doi: 10.2307/3094873

Reinstein, D. A. \& Snyder, C. M. (2005). The influence of expert reviews on consumer demand for experience goods: A case study of movie critics. The Journal of Industrial Economics, 53(1), 27-51. doi: 10.1111/j.0022-1821.2005.00244.x

Salmon, S. J., De Vet, E., Adriaanse, M. A., Fennis, B. M., Veltkamp, M. \& De Ridder, D. T. (2015). Social proof in the supermarket: Promoting healthy choices under low self-control conditions. Food Quality and Preference, 45, 113-120. doi: 10.1016/j.foodqual.2015.06.004

Serra, A. \& Salvi, F. (2014). New consumer behavior: A review of research on eWOM and hotels. International Journal of Hospitality Management, 36, 41-51. doi: 10.1016/j.ijhm.2013.08.007

Shim, S., Eastlick, M. A., Lotz, S. L. \& Warrington, P. (2001). An online prepurchase intentions model: The role of intention to search. Journal of Retailing, 77, 397416. doi: 10.1016/S0022-4359(01)00051-3

Sparks, B. A. \& Browning, V. (2011). The impact of online reviews on hotel booking intentions and perception of trust. Tourism Management, 32(6), 1310-1323. doi: 10.1016/j.tourman.2010.12.011

Tuominen, P. (2011). The Influence of TripAdvisor Consumer-Generated Travel Reviews on Hotel Performance. 19th annual Frontiers in Service Conference. University of Hertfordshire. (https://goo.gl/gm3zXc) (2017-1-22).

Vermeulen, I.E. \& Seegers, D. (2009). Tried and tested: The impact of online hotel reviews on consumer consideration. Tourism Management, 30, 23-127. doi: 10.1016/j.tourman.2008.04.008

Yacouel, N. \& Fleischer, A. (2010). The Role of Cybermediaries in the Hotel Market. The Hebrew University of Jerusalem.

Ye, Q., Law, R. \& Gu, B. (2009). The impact of online user reviews on hotel room sales. International Journal of Hospitality Management, (28), 180-182. doi: 10.1016/j.ijhm.2008.06.011

Zhu, F. \& Zhang, X. (2010). Impact of online consumer reviews on sales: The moderating role of product and consumer characteristics. Journal of Marketing, 74(2), 133-148. doi: 10.1509/jmkg.74.2.133 